\title{
Using Arduinos to Transition a Bioinstrumentation Lab to Remote Learning
}

\author{
Abiche H. Dewilde (i) and Yanfen Li (D) \\ Department of Biomedical Engineering, University of Massachusetts Lowell, 1 University Ave, Lowell, MA 01876, USA
}

(Received 30 June 2020; accepted 13 November 2020; published online 30 November 2020)

\section{CHALLENGE STATEMENT}

Bioinstrumentation is a required course in more than $75 \%$ of accredited BME programs. ${ }^{1}$ Like many other institutions, the bioinstrumentation lab at the University of Massachusetts Lowell (UMass Lowell) is a junior level course designed to provide the students with fundamental understanding of electrical circuits, circuit components, and bioinstrument design. The course meets weekly and the students progress through a series of labs where they build, test, and troubleshoot basic biosensor circuits. Example labs include building voltage dividers, active and passive filters, electrocardiogram (ECG), and electromyograph (EMG). Typically, these labs use a variety of equipments including function generators and oscilloscopes. At UMass Lowell, we utilize the iWorx BIK-TA BioInstrumentation Physiology Teaching Kit. ${ }^{2}$ For the latter half of the semester, the lab course culminates in a team design project. For these projects, the students are typically placed into groups of 2-3 students to create an instrumentation device. The groups must propose, design, and execute a self-directed project to convert a raw biological signal into physiologically relevant data. This bioinstrument is expected to detect a signal with a chosen biosensor, modify this analog signal, and then supply a digital output. The groups must also conduct data analysis and then report their findings with an oral presentation and a scientific report.

UMass Lowell transitioned abruptly to remote learning during the spring semester of 2020 during which students were preparing to conduct their last lab (EMG) and begin their final semester project in groups

Address correspondence to Yanfen Li, Department of Biomedical Engineering, University of Massachusetts Lowell, 1 University Ave, Lowell, MA 01876, USA. Electronic mail: Yanfen_Li@uml. edu of 2-3 students. Several challenges were presented in transitioning this bioinstrumentation lab - with a big project - to online. First, students were working from home without access to the equipment available from campus. Without access to sensors, circuit boards, function generators, and oscilloscopes, it was almost impossible for students to gain the hands-on experience of designing and building their own circuits. Second, the students had also lost access to each other. This made it difficult to facilitate group work and to provide support as students worked on their projects.

\section{NOVEL INITIATIVE}

Since the students no longer had access to the iWorx teaching kits and biosensors available in the lab, alternatives were explored which allowed students to still build and test circuits from home. It was determined that a cheap alternative is for each student to purchase the ELEGOO UNO Project Basic Starter Kit with Tutorial and UNO R3 Compatible with Arduino Integrated Development Environment (IDE) $(\sim \$ 18){ }^{3}$ The Arduino systems are low cost systems, agnostic towards a student's operating system availability, and have previously been used in STEM education, and in biomedical engineering. ${ }^{46}$ Each student purchased this kit online and the kit was shipped to their homes within 2-5 days. The students were to be reimbursed for the cost if they chose to return the kit to campus.

Up to this point the students had been using a different interface and software, thus a quick transition plan needed to be created for the students to familiarize themselves with the Arduino platform. The students were tasked with learning this new platform while simultaneously researching their project topic. To help the students learn the new Arduino environ- 
ment, Autodesk Tinkercad $\odot$ circuits was used. ${ }^{7}$ Tinkercad $\subset$ circuits is a software that simulates electrical circuits and components which has previously been used in science instruction. ${ }^{8,9}$ The Tinkercad(C) circuits feature enabled the students to start building and simulating virtual Arduino circuits on their platform. Tinkercad $\subset$ has created a series of YouTube instructional videos that were perfect for introduction to the system. A class was setup on the Tinkercad (C) platform and the students were instructed to complete all the Learn Arduino with Tinkercad@ Circuits YouTube videos. ${ }^{10}$

The students were also taught about the Zoom video conferencing, which allowed them to communicate with their group members, TAs, and instructor. Before the first Zoom meeting, the students were advised to have open-source Arduino Software (IDE) installed on their computer. Each week the students attended their regularly scheduled lab via Zoom. The lab would start with a general update regarding where the student should be in their project and what was expected of them in the coming weeks. This often involved a demo of a model project created by the instructor, and the availability of pre-recorded videos in specific topics needed for the project. Topics included citation management, report design, data analysis, and creating a professional PowerPoint. Once these points were covered, the instructor used Zoom to assign each group to a "breakout room." Then TAs and instructors were added to these breakout rooms by priority as determined by questions from the groups, the instructor's relevant background knowledge of the projects, project status, and teaching assistants' (TA) background.

The students could then start working on their project together. Students used this time to talk to each other about the project, assign portions to the project, and do concurrent builds using the Arduino kit and Zoom in group breakout rooms. There were frequent check-ins from instructor and TAs to answer questions. Answers to common question was announced to all students and provided on blackboard.

Students were able to work through most problems during regular course time and requested additional time with instructor as needed. These additional meetings became more frequent towards the end of the semester when final debugging of their biosensor was occurring before their presentation. An Excel spreadsheet was made to aid monitoring of project progress which included the project concept, components they were working with, and notes on progress or issues. This also facilitated pairing of groups with TAs and kept track of hours.

All students used the same components as provided by the instructor. This is vital to avoid shield model version confusion, and reduces the instructors' need to familiarize themselves with more components. Therefore, multi packs of components were often picked from Sparkfun, Digikey, Adafruit or Amazon an$d$ then distributed. One component of each type was retained with the instructor for build assistance.

With all the students working on the same base Arduino UNO module, Arduino IDE software, and components, the instructors could easily mimic students' builds for troubleshooting. Students would upload their schematics and codes to Autodesk Tinkercad $\odot$ circuits. Then, all group members and instructors would download the same code, upload it to their device, and check the response. This allowed everyone to start at the same build before testing and troublshooting in real time while on zoom.

\section{REFLECTION}

\section{Instructor Reflections}

In project guidelines, more latitude was provided in terms of biological signals measured to allow for the unique scenario of at home research. In the original plan of the class, once the groups completed the project, they were supposed to present it to their peers. In the remote format, the groups created a power point presentation with embedded videos which they recorded using Zoom. This allowed for all members of the group to participate in the presentation. The link to the presentation was provided to the instructor, either as a Zoom recording or as a YouTube recording. With permission from students, sample presentation videos are available below. A Google Sheets survey (provided in the supplementary materials) was created with the link of every presentation and the grading rubric allowing the students to score their peers. Finally, they were asked which project they liked best and why. This survey was also used by the instructor and TAs and all scores, including peer scores, were used in the final grade. Slightly more weight was applied to the instructor's and TAs' scores.

The projects chosen by students were more ambitious than those seen in previous years. In the previous year, projects included night lights or fire alarms with the most ambitious being a photoplasmograph. However, most of the groups were not able to provide a working prototype. Interestingly, with the remote learning using the Arduino system, the level of complexity rose significantly. The simplest projects involved rotating a plant to optimize light exposure, which was first designed in Tinkercad (C) circuits (Fig. 1) and subsequently built and tested. Examples of more ambitious projects included posture alert systems to improve worker ergonomics, ${ }^{11}$ an EEG to detect patient emotions for non-verbal autistic patients, ${ }^{12}$ a prosthetic hand that incorporated EMG to 


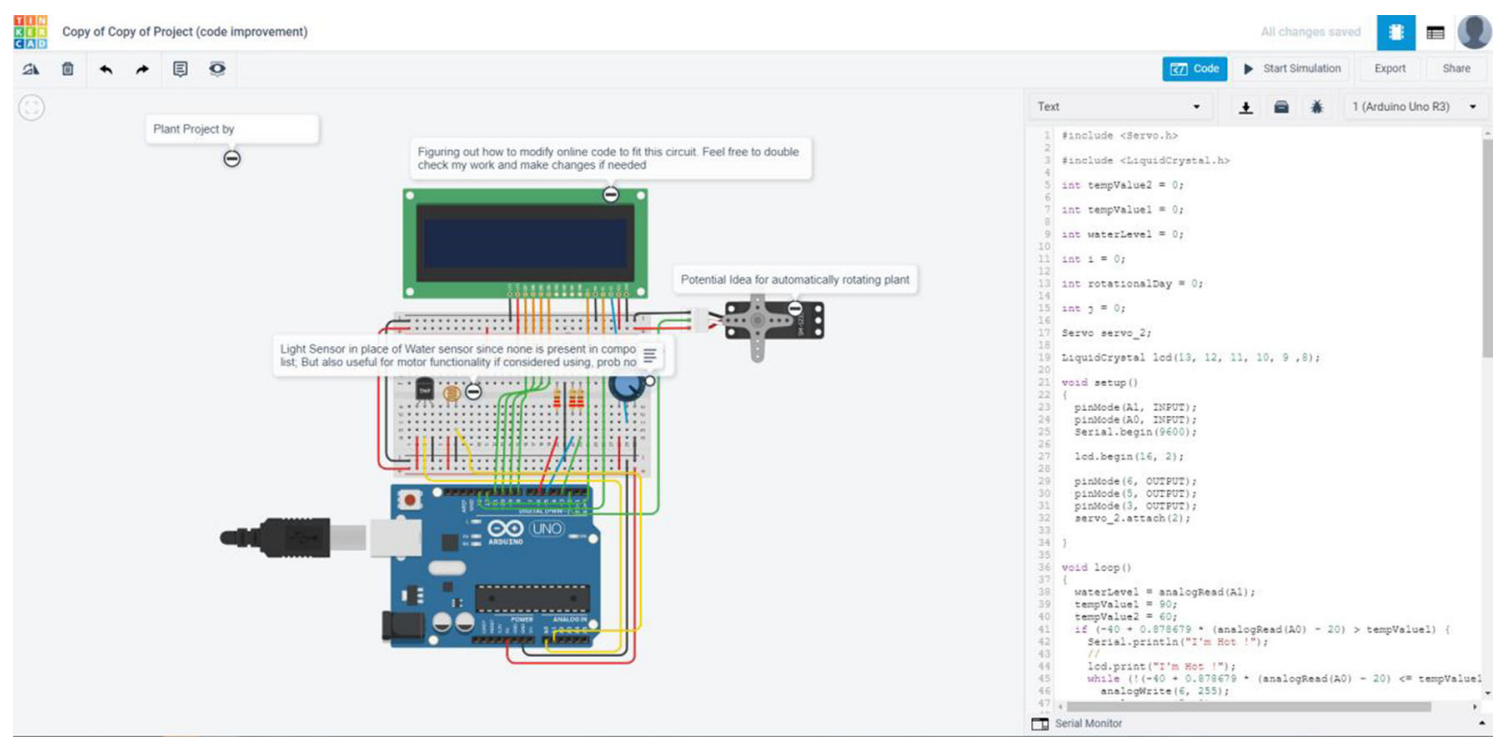

FIGURE 1. Tinkercad circuit representation of one student group's project to orient a plant towards the light to improve growth, with the final goal of improving crop yield to decrease environmental impact of agriculture.

move, ${ }^{13}$ and an epileptic seizure detection system. ${ }^{14}$ All of these projects were successful in obtaining basic data, however since the scale of the project was very ambitious, additional improvements were needed.

One potential challenge of this method is the range of different components used and the need to find relevant product information. It was useful to create a folder with all product information sheets and notes on known issues to be shared with students and TAs. Example issues included different pinout orders from those used in class, special power requirements, etc.

An unforseen benefit of the new format was that every group created a video recording which allowed students across different section to watch all the presentations. In addition, these recordings have become a departmental resource for students in future courses and for recruiting.

\section{Student Feedback}

During the final survey, student feedback was also solicited to determine what students liked and did not like about the remote labs. The students like having their own Arduino UNO system to work on, because it allows them to work at their own pace and repeat specific exercises to make sure they understand the concept. They even mentioned that they would have liked to have Arduinos at the beginning of the semester, instead of when it was introduced during the transition. Some students felt like switching midway created a larger workload to have to re-learn a new data acquisition system though this should not be an issue if planned for the beginning of the semester.
The students also stated that using Autodesk Tinkercad $\odot$ circuits allowed the group to work on the same project and code and test ideas before they built the circuit physically. It also allowed them to easily share schematics and code with each other. However, they still wanted to be able to meet with their partners in small groups to troubleshoot during the project, especially in the final week when working on the prototype. Being able to present together would also be optimal since some of them had issued with IT equipment-making recording on Zoom unreliable and reduced the audio quality of the presentation. With the quarantine restrictions being eased, some inperson meeting is a possibility, as well as a face to face meeting with the instructor as needed.

Overall, the adaptation of an Arduino system for an at-home bioinstrumentation lab was successful. While it might be more cumbersome for the instructor to coordinate purchasing of Arduinos and circuit components, the overall result was positive. With the Zoom platform, the instructor was able to effectively teach the course topics and aid with student troubleshooting. Students also enjoyed being able to do hands on activities at home and some students mentioned that they enjoyed this system better since they were able to work on it by themselves.

When talking to the students to obtain their thoughts about what part of the changes they would like to continue to use after the COVID-19 pandemic, the overall impressions were positive. They liked having their own system that they could use at home to practice, and access to tutorials that could assist them. They also stated that there were a lot of resources that 
they could access when designing their projects on an Arduino-based system, and that components came with libraries of basic code. They stated that they felt that this would prepare them for industry by teaching them how to research and implement commercially available components. Some students reported that this experience with the Arduino systems and the "Maker" experience has helped them during their interviewing process, and in securing a job post graduation.

Given this feedback, if the class is taught remotely, the Arduino system will be the base system for labs. However, given the success and simplicity of this system, we plan to introduce the Arduino system to students at the beginning of the semester before transitioning to the more complex iWorx system. This will allow them the creative freedom that an Arduino based project provides that was previously not possible.

\section{ELECTRONIC SUPPLEMENTARY MATERIAL}

The online version of this article (https://doi.org/10. 1007/s43683-020-00042-9) contains supplementary material, which is available to authorized users.

\section{AUTHOR CONTRIBUTIONS}

$\mathrm{AD}$ was the lead in writing the paper as well as the instructor for the class that had to transition to remote learning. YL previously taught the class and also contributed to the writing of the manuscript.

\section{FUNDING}

None.

\section{DATA AVAILABILITY}

None.

None.

\section{CONFLICTS OF INTEREST}

ETHICS APPROVAL

None.

\section{INFORMED CONSENT}

Students whose projects were used were asked permission to share their presentations.

\section{REFERENCES}

${ }^{1}$ David WGP, Robert AL. Similarities and differences in undergraduate biomedical engineering curricula in the united states. In: ASEE conferences, Indianapolis, Indiana. ${ }^{2}$ iworx. Bik-ta bioinstrumentation physiology teaching kit. iworx.

${ }^{3}$ Elegoo uno project basic starter kit with tutorial and uno r3 for arduino. 2020. https://www.elegoo.com/product/eleg oo-uno-project-basic-starter-kit-with-tutorial-and-uno-r3-f or-arduino/. Accessed 30 June 2020

${ }^{4}$ More missing the boat - arduino, raspberry pi, and small prototyping boards and engineering education needs them. In: 2015 IEEE frontiers in education conference (FIE), 2124 Oct 2015, 2015.

${ }^{5}$ Designing arduino electronic shields: Experiences from secondary and university courses. In: 2017 IEEE global engineering education conference (EDUCON), 25-28 April 2017, 2017.

${ }^{6}$ Puente ST, Úbeda A, Torres F. E-health: biomedical instrumentation with arduino. IFAC-PapersOnLine. 2017:50(1):9156-61.

${ }^{7}$ Autodesk tinkercad circuits. 2019. https://www.tinkercad.c om/dashboard.

${ }^{8}$ Smetana LK, Bell RL. Computer simulations to support science instruction and learning: a critical review of the literature. Int J Sci Educ. 2012;34(9):1337-70.

${ }^{9}$ Tinkercad and codeblocks in a summer course: an attempt to explain observed engagement and enthusiasm. In: 2019 IEEE blocks and beyond workshop (B\&B), 18-18 Oct 2019, 2019

${ }^{10}$ Autodesk Tinkercad Learn arduino with tinkercad circuits. 2018. https://www.youtube.com/playlist?list $=$ PLV6 $\mathrm{cmKv}$ nKRs5geApVORPW79U6s3wpa0Ht. Accessed 5 June 2020.

${ }^{11}$ Doherty E, Dulaney A. Arduino posture alert system. 2020. https://uml.ensemblevideo.com/Watch/Arduino_Video. Accessed 5 June 2020.

${ }^{12}$ Fiora L, Dickey S. Mood waves. 2020. https://uml.ensemb levideo.com/Watch/Mood_Waves Accessed 5 June 2020.

${ }^{13}$ Mulry A, Miller T. Muscle controlled prosthetic hand. 2020. https://uml.ensemblevideo.com/Watch/Final_Project Accessed 5 June 2020.

${ }^{14}$ Goncalves RC, Kabulo G, Haodong L. Epileptic seizure detection. 2020. https://uml.ensemblevideo.com/Watch/Ep ileptic_Seizure_Detection Accessed 5 June 2020.

Publisher's Note Springer Nature remains neutral with regard to jurisdictional claims in published maps and institutional affiliations. 